\title{
Immature Granulocyte Count and Delta Neutrophil Index as New Predictive Factors for Axillary Metastasis of Breast Cancer
}

Mehmet Bugra Bozan ${ }^{1}$, Fatih Mehmet Yazar ${ }^{1}$, Ilhami Taner Kale ${ }^{1}$, Sezgin Topuz ${ }^{1}$, Ayse Azak Bozan² and Omer Faruk Boran ${ }^{3}$

\begin{abstract}
${ }^{1}$ Department of General Surgery, Kahramanmaras Sutcu Imam University, Kahramanmaras, Onikisubat, Turkey
${ }^{2}$ Department of Anesthesiology and Reanimation, Necip Fazil State Hospital, Kahramanmaras, Onikisubat, Turkey

${ }^{3}$ Department of Anesthesiology and Reanimation, Kahramanmaras Sutcu Imam University, Onikisubat, Turkey
\end{abstract}

\begin{abstract}
Objective: To determine the diagnostic value of preoperative immature granulocyte (IG) count and delta neutrophil index (DNI) level before clinical detection of axillary lymph node metastasis.

Study Design: Cohort study.

Place and Duration of Study: Department of General Surgery, Kahramanmaras Sutcu Imam University, Onikisubat, Turkey from February 2015 to February 2020.

Methodology: Patients older than 18 years and operated for breast pathologies in the study period were evaluated retrospectively. Patients without axillary or distant organ metastasis, and who did not receive neoadjuvant chemotherapy were examined by dividing them into two groups as pathologically non-metastatic axilla (Group NM) and metastatic axilla (Group M). They were retrospectively evaluated for DNI, IG, white blood cell count (WBC), neutrophil/lymphocyte ratio (NLR), platelet/lymphocyte ratio (PLR) and mean platelet volumes (MPV).

Results: All of the 83 patients, who met the inclusion criteria, were females (100\%). Forty-six patients $(55.42 \%)$ were in the Group-NM and $37(44.58 \%)$ were in Group-M. Statistically significant difference was observed between the groups in terms of WBC, NLR, PLR, MPV, DNI and IG count $(p<0.05)$, while there was no difference in age $(p=0.862)$. As a result of the univariate and multivariate analysis, WBC, NLR, PLR, MPV, DNI and IG count were determined as predictive factors. The discriminatory power of the DNI for diagnosing clinically negative pathologically positive axillary metastasis of breast cancer at the cut-off value $\geq 0.35 \%$ (ARUC:0.903; 95\% confidence interval [CI]: $0.84-0.967$ ) showed $86.5 \%$ sensitivity, $80.4 \%$ specificity, positive predictive value (PPV) $86.5 \%$, negative predictive value (NPV) $80.4 \%$. The discriminatory power of the IG count for diagnosing clinically negative pathologically positive axillary metastasis of breast cancer, at the cut-off value $\geq 25 / \mathrm{mm}^{3}$ (ARUC:0.976; $95 \% \mathrm{Cl}: 0.953-1.000$ ) showed $100 \%$ sensitivity, $82.6 \%$ specificity, $100 \%$ PPV, and $82.6 \%$ NPV.

Conclusion: DNI and IG count may be new predictive factors with high sensitivity and specificity in detecting axillary metastasis of breast cancer.
\end{abstract}

Key Words: Delta neutrophil index, Immature granulocyte count, Neutrophil lymphocyte ratio, Breast cancer, Axillary metastasis.

How to cite this article: Bozan MB, Yazar FM, Kale IT, Topuz S, Bozan AA, Boran OF. Immature Granulocyte Count and Delta Neutrophil Index as New Predictive Factors for Axillary Metastasis of Breast Cancer. J Coll Physicians Surg Pak 2022; 32(02):220-225.

\section{INTRODUCTION}

Breast cancer ranks first among cancer types seen in women in our country and all over the world, and second after lung cancer in cancer-related deaths. ${ }^{1,2}$ Despite the recent increase in its incidence, mortality has decreased due to early diagnosis and advances in neo-adjuvant therapy. ${ }^{2,3}$

Correspondence to: Dr. Mehmet Bugra Bozan, Department of General Surgery, Kahramanmaras Sutcu Imam University, Kahramanmaras, Onikisubat, Turkey

E-mail: bbozan@yahoo.com

Received: January 08, 2021; Revised: May 10, 2021;

Accepted: June 07, 2021

DOI: https://doi.org/10.29271/jcpsp.2022.02.220
Classically, lymph node status, tumor size, histological type, grade, age, and ethnicity are prognostic factors for this type of cancer. ${ }^{4}$

Bone marrow activation results from malignancies and inflammation. Tumor-related inflammation has gained importance in each stage of tumorigenesis. Host-dependent systemic inflammatory response has been found to be effective in carcinogenesis, tumor development and progression..$^{2-4}$ Inadequately controlled oruncontrolled inflammatory activity may be responsible formalignant transformation. ${ }^{5,6}$ Inflammatory cell stimulation occurs in lymph node metastasis and distant organ metastasis like primary tumor. ${ }^{7}$ Generally, lymphocytes and monocytes are essential immunoinflammatory cells. ${ }^{2}$ As the cornerstone of the adaptive immune system, lymphocytes inhibit tumor cell proliferation, migration and destroy metastatic 
lesions. ${ }^{3,6}$ On the other hand, tumor-induced neutrophils may accelerate tumor metastasis. ${ }^{2}$ Many studies have examined the relationship among the ratios of different cell types (such as the platelet-lymphocyte ratio (PLR), and the neutrophil-lymphocyte ratio (NLR) in malignant tumors. ${ }^{3,8}$ Apart from these, mean platelet volume (MPV), which can be automatically studied in routine blood counts and shows platelet activation, has been used to show tumor activity in breast, stomach, colon and ovarian cancers. ${ }^{9}$ These markers are reproducible, easily applicable, inexpensive, and have no additional cost; therefore, the inflammatory response can be easily monitored. ${ }^{10}$

Recent studies have identified the delta neutrophil index (DNI), which indicates the percentage of immature granulocytes (IG) in peripheral blood due to increased bone marrow activation in inflammatory conditions. It is based on counting granulocyte precursor cells under microscope..$^{5}$

The IG fractions included promyelocytes, myelocytes and metamyelocytes, but not band neutrophils or myeloblasts. Moreover, the DNI is the ratio of the IG count to the white blood cell count. ${ }^{5,11}$ With the technological developments, IG count and DNI can automatically be evaluated from complete blood count parameters in automated systems. A cancer study on these parameters, which were previously studied in inflammatory processes such as sepsis, cardiovascular events, acute appendicitis, and urolithiasis, is only available in a study that distinguishes thyroid cancers from benign nodules. ${ }^{5,12}$

The objective of this study was to determine the diagnostic value of preoperative IG number and DNI level before clinical detection of axillary lymph node metastasis, which plays an important role in the prognosis of breast cancer, and to compare these parameters with other routine inflammation markers such as white blood cell count (WBC), MPV, NLR and PLR.

\section{METHODOLOGY}

Ethical approval was obtained from Kahramanmaras Sutcu Imam University, Clinical Research Ethics Committee (Date: 13.05.2020; Session No. 2020/09; Decision No. 19). Patients, who were older than 18 years and operated for breast pathologies in Kahramanmaras Sutcu Imam University, Department of General Surgery between February 2015 and February 2020, were evaluated in this retrospective cohort study. All procedures performed in studies, involving human participants, were in accordance with the ethical standards of the institutional and/or national research committee and with the 1964 Declaration of Helsinki, and its later amendments or comparable ethical standards. Patient data were obtained from patient epicrisis forms and preoperative laboratory; and postoperative pathology results recorded in the computer system. Demographic data of the patients, routine blood tests, preoperative tumor size, presence of axillary lymph node metastasis and distant organ metastasis, presence of pathological axillary lymph node metastasis in the postoperative period were evaluated.
Inclusion criteria were: patients diagnosed with invasive breast cancer (invasive ductal carcinoma, invasive lobular carcinoma, mixed invasive ductal lobular carcinoma, mucinous carcinoma, medullary carcinoma, micropapillary carcinoma), who did not have clinically axillary metastasis and axillary neo-adjuvant chemotherapy/radiotherapy; buthavepostoperativepathologically positive or negative axillary metastasis. Patients, who underwent surgery for benign breast disease (such as fibroadenomas, granulomatous mastitis, hamartomas); patients with breast malignancies other than invasive breast carcinoma (ductal carcinoma in situ, fibrosarcomas, neuroendocrine tumors, myofibroblastomas, phylloides tumors); patients with clinically axillary metastasis or with distant organ metastasis in the preoperative period; patients who received neo-adjuvant chemotherapy/radiotherapy in the preoperative period, and patients with insufficient data, were excluded from the study.

In the preoperative period, patients without axillary metastases and who did not receive neo-adjuvant treatment were examined by dividing them into two groups as pathologically non-metastatic (Group-NM) and had tumor metastasis in $\geq 1$ axillary LN (Group-M).

WBC count, neutrophil count, lymphocyte count, platelet count, MPV, IG count and DNI were measured using an automated hematological analyser (XN 3000; Sysmex Corp., Kobe, Japan) from blood samples obtained within the last month of surgery. This device automatically measures DNI using two independent leukocyte counting methods: myeloperoxidase and lobularite/nuclear density-channel. ${ }^{12}$ NLR and PLR were manually calculated from the complete blood cell results.

IBM-statistical package for social sciences (SPSS) version 20 was used for statisticalanalysis. The distribition of data was evaluated by Shapiro-Wilk test. While student t-test or MannWhitney U-test was used in the evaluation of continous variables, according to the conformity of the data to normal distribution, Chi-square test was used in the evaluation of categorical data. Multivariate analysis was performed with parameters found to be significant in the evaluation of univariate analysis; and predictive values of preoperative blood parameters were calculated. ROC analysis was performed to evaluate the effectiveness of WBC, NLR, PLR, DNI, IG number and MPV values, and sensitivity, specificity values and cut-off values were obtained. Numerical values were expressed as mean \pm standard deviation (minimum - maximum values) or median $\left(25^{\text {th }}-75^{\text {th }}\right.$ percentiles), according to distrubition of normality; and categorical values as percentage(\%). A $p$-value of $<0.05$ was considered as statistically significant. The power analysis of the study was conducted with the G-Power 3.0.10 programming system. In the estimation of power analysis and sample size, for a power of $0.8, \alpha=0.1$ and an effect size of 0.5 in the evaluation of two independent groups 80 patients were required.

\section{RESULTS}

All patients were females (100\%). Forty-six of these 83 patients (55.42\%) were in Group-NM, while 37 (44.58\%) in Group-M 
(Figure 1). Statistically significant difference was observed between the groups in terms of blood parameters (WBC, NLR, PLR, MPV, DNI and IG count, $p<0.05$ ), while there was no difference between Group-NM and Group-M in terms of age ( $p=$ 0.862 , TableI).

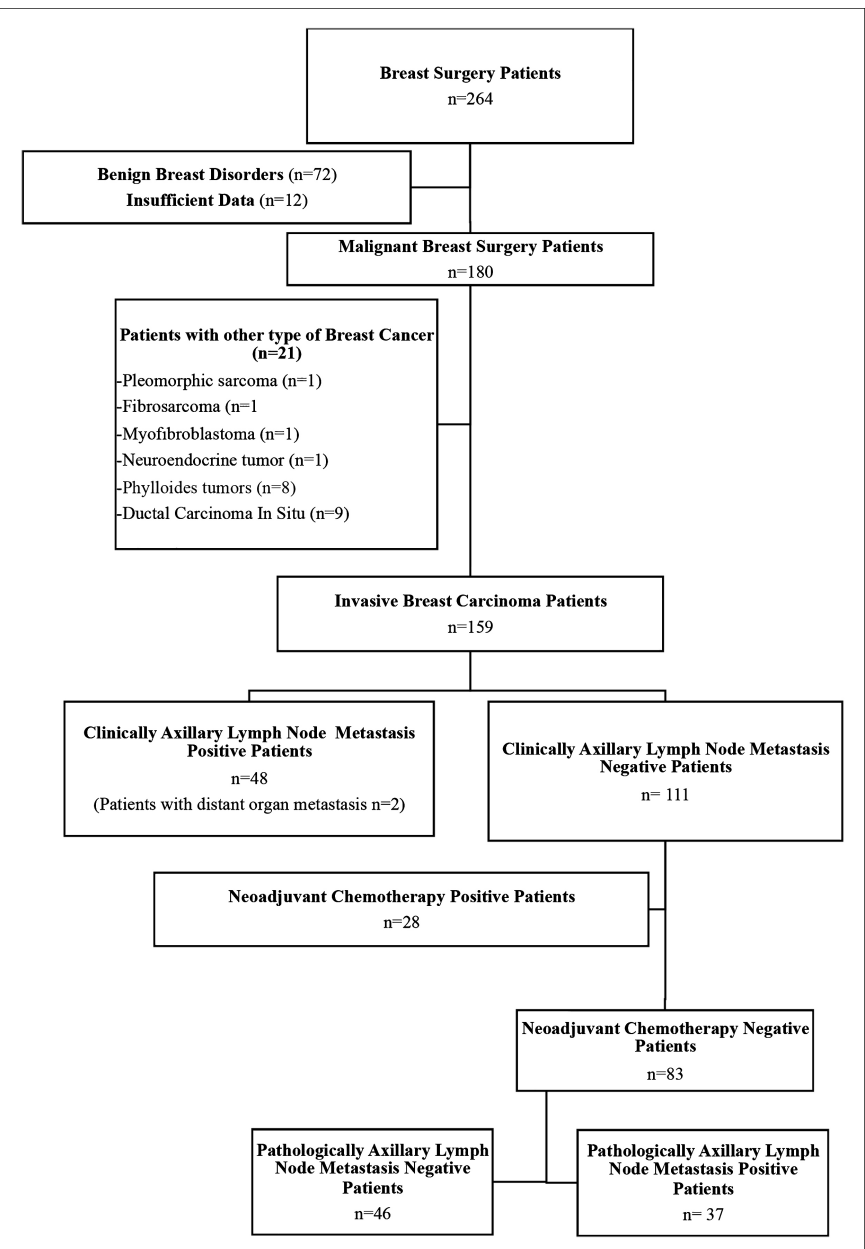

Figure 1: Flow chart of the study.

Univariate analysis showed that age $(p=0.937 ; F: 0.006$; $\mathrm{OR}=0.000$ ) was not associated with the presence of axillary metastasis pathologically in patients with clinically negative axilla. WBC, NLR, PLR, MPV, DNI and IG count were also associated with development of axillary lymph node metastasis in follow-up in univariable and multivariable analysis (Tablell).

The discriminatory power of the DNI for diagnosing clinically negative pathologically positive axillary metastasis of breast cancer, for the cut-off value $\geq 0.35 \%$ (ARUC: $0.903 ; 95 \%$ confidence interval $[\mathrm{Cl}]: 0.84-0.967)$, showed that sensitivity was $86.5 \%$, specificity was $80.4 \%$, positive predictive value (PPV) was $86.5 \%$, negative predictive value (NPV) was $80.4 \%$. The discriminatory power of the IG count for diagnosing clinically negative pathologically positive axillary metastasis of breast cancer, for the cut-off value $\geq 25 / \mathrm{mm}^{3}$ (ARUC:0.976; $95 \%$ $\mathrm{Cl}: 0.953-1.000$ ) showed that sensitivity was $100 \%$, specifity was $82.6 \%$, PPV was $100 \%$, and NPV was $82.6 \%$ (Table III, Figure 2).
Table I: Demographic data and the preoperative blood sample results of thestudy.

\begin{tabular}{|c|c|c|c|}
\hline & \multicolumn{2}{|c|}{ Clinically Axillary LN Metastasis (-) } & \multirow[b]{2}{*}{ p-value } \\
\hline & $\begin{array}{c}\text { Group NM } \\
\text { (Pathologically } \\
\text { Axillary LN } \\
\text { Metastasis (-)) } \\
\text { [Mean } \pm \text { S.D } \\
\text { (Minimum - } \\
\text { Maximum) } / \text { Median } \\
\left(25^{\text {th }}-7^{\text {th }}\right. \\
\text { percentiles)] }\end{array}$ & $\begin{array}{c}\text { Group M } \\
\text { (Pathologically } \\
\text { Axillary LN } \\
\text { Metastasis (+)) } \\
\text { [Mean } \pm \text { S.D (Minimum } \\
\text { - Maximum) /Median } \\
\left(25^{\text {th }}-75^{\text {th }}\right. \\
\text { percentiles)] }\end{array}$ & \\
\hline Age $\left(\right.$ year) ${ }^{b}$ & $52(45.0-64.5)$ & $51(43.5-63.5)$ & 0.862 \\
\hline $\begin{array}{l}\text { Preoperative } \\
\text { Tumor Size } \\
(\mathrm{mm})^{b}\end{array}$ & $20(16-35)$ & $26(20-45)$ & 0.277 \\
\hline $\mathrm{WBC}\left(/ \mathrm{mm}^{3}\right)^{\mathrm{a}}$ & $\begin{array}{l}7073.04 \pm 970.31 \\
(5430-9090)\end{array}$ & $\begin{array}{c}9006.76 \pm 1029.83 \\
(7400-11510)\end{array}$ & $<0.001 *$ \\
\hline$N L R^{b}$ & $1.91(1.57-2.26)$ & $3.24(2.81-4.17)$ & $<0.001^{*}$ \\
\hline $\mathrm{PLR}^{\mathrm{b}}$ & $113.33(86.79-142.22)$ & $182.18(155.13-218.46)$ & $<0.001^{*}$ \\
\hline MPV $(f L)^{b}$ & $9.6(8.9-10.03)$ & $10.8(10.1-11.65)$ & $<0.001^{*}$ \\
\hline DNI $(\%)^{b}$ & $0.3(0.2-0.3)$ & $0.5(0.4-0.65)$ & $<0.001^{*}$ \\
\hline IG count $\left(/ \mathrm{mm}^{3}\right)^{\mathrm{b}}$ & $20(10-20)$ & $40(30-60)$ & $<0.001^{*}$ \\
\hline \multicolumn{4}{|c|}{$\begin{array}{l}\text { *p<0.05, a: According to student t-test; b: Acording to Mann Whitney U- } \\
\text { Test. WBC: White blood cell count; NLR: Neutrophil to lymphocyte ratio; PLR: } \\
\text { Platelet to lymphocyte ratio; DNI: Delta neutrophil index; IG: Immature } \\
\text { granulocyte; LN: Lymph Node. }\end{array}$} \\
\hline
\end{tabular}

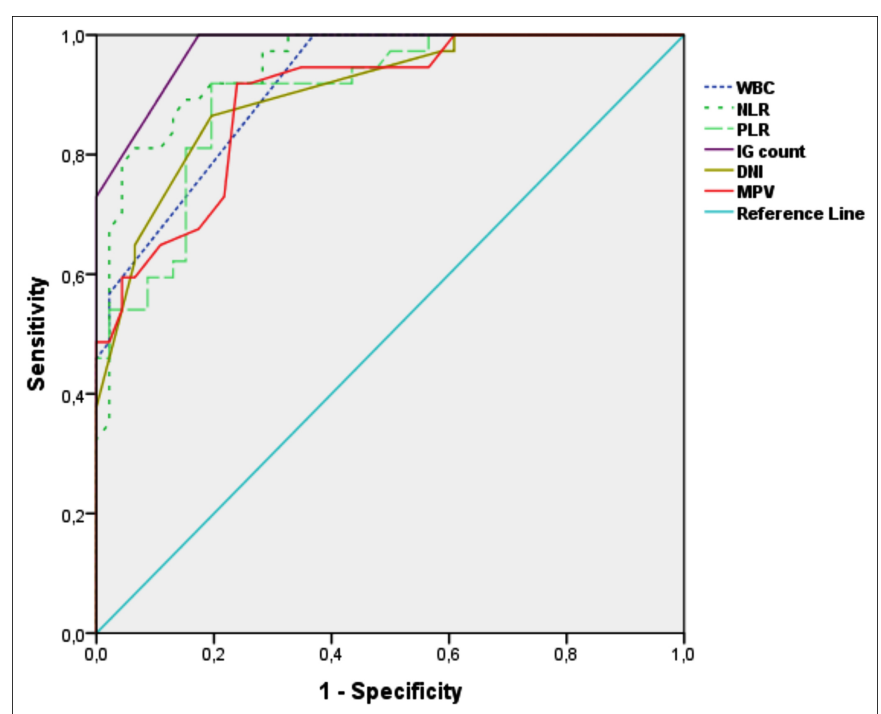

Figure 2: ROCcurve of inflammatory markers fordiagnosing clinicallyaxillary lymphnode metastasis negative and pathologically axillary lymphnode metastasis positive invasive breast cancer patients (WBC: White blood cell count; NLR: Neutrophil to lymphocyte ratio; PLR: Platelet to Iymphocyte ratio; IG: Immature granulocyte; DNI: Delta neutrophil index; MPV: Mean platelet volume).

\section{DISCUSSION}

Tumor-induced noninfectious inflammatory response is important in the prognosis and clinical outcomes of patients; and for this purpose, inflammation-based scoring systems derived from complete blood counts such as NLR, PLR, MPV are used as an indicator of inflammatory response to the tumor. ${ }^{3,5,13}$ Low lymphocyte counts indicate an inadequate immune response for many types of cancer, while Tlymphocytes enable tumor-associated antigens to destroy cancercells. ${ }^{3,14,15}$ 
Table II: Multivariante analyses results of inflammatory markers for prediction of pathologically axillary lymph node metastasis positivity for clinically axillary lymph node metastasis negative patients.

\begin{tabular}{|c|c|c|c|c|c|}
\hline & \multirow{2}{*}{ Odds Ratio } & \multirow{2}{*}{ B-value } & \multirow{2}{*}{ p-value } & \multicolumn{2}{|c|}{ \%95 Coefficient Interval } \\
\hline & & & & Lower Bound & Upper Bound \\
\hline WBC & 0.966 & 7073.043 & $<0.001^{*}$ & 6780.501 & 7365.586 \\
\hline NLR & 0.676 & 1.924 & $<0.001 *$ & 1.630 & 2.218 \\
\hline PLR & 0.767 & 116.973 & $<0.001^{*}$ & 102.713 & 131.233 \\
\hline MPV & 0.984 & 9.413 & $<0.001 *$ & 9.151 & 9.675 \\
\hline DNI & 0.663 & 0.270 & $<0.001^{*}$ & 0.227 & 0.312 \\
\hline IG count & 0.481 & 18.696 & $<0.001 *$ & 14.402 & 22.990 \\
\hline
\end{tabular}

Table III: ROC curve analysis of inflammatory markers for diagnosing clinically axillary negative and pathologically axillary positive breast cancer patients.

\begin{tabular}{|c|c|c|c|c|c|c|c|c|c|}
\hline & \multirow[t]{2}{*}{ ARUC } & \multirow[t]{2}{*}{ PPV (\%) } & \multirow[t]{2}{*}{ NPV (\%) } & \multirow[t]{2}{*}{ Cut-off value } & \multirow[t]{2}{*}{ Sensitivity (\%) } & \multirow{2}{*}{$\begin{array}{l}\text { Specivitiy } \\
(\%)\end{array}$} & \multicolumn{2}{|c|}{$\begin{array}{c}\text { Asymptotic } 95 \% \text { Confidence } \\
\text { Interval }\end{array}$} & \multirow[t]{2}{*}{ p-value } \\
\hline & & & & & & & Lower Bound & Upper Bound & \\
\hline WBC $\left(/ \mathrm{mm}^{3}\right)$ & 0.913 & 81.1 & 78.3 & $\geq 7865$ & 81.1 & 78.3 & 0.857 & 0.97 & $<0.001^{*}$ \\
\hline NLR & 0.947 & 91.9 & 80.4 & $\geq 2.29$ & 91.9 & 80.4 & 0.903 & 0.991 & $<0.001^{*}$ \\
\hline PLR & 0.900 & 91.9 & 80.4 & $\geq 145$ & 91.9 & 80.4 & 0.836 & 0.964 & $<0.001^{*}$ \\
\hline MPV (fL) & 0.894 & 91.9 & 76.1 & $\geq 10.05$ & 91.9 & 76.1 & 0.827 & 0.96 & $<0.001^{*}$ \\
\hline IG Count $\left(/ \mathrm{mm}^{3}\right)$ & 0.976 & 100 & 82.6 & $\geq 25$ & 100 & 82.6 & 0.953 & 1.000 & $<0.001^{*}$ \\
\hline DNI (\%) & 0.903 & 86.5 & 80.4 & $\geq 0.35$ & 86.5 & 80.4 & 0.84 & 0.967 & $<0.001 *$ \\
\hline
\end{tabular}

${ }^{*} p<0.05$. ARUC: Area under curve; PPV: Positive predictive value; NPV: Negative predictive value; WBC: White blood cell count; NLR: Neutrophil to lymphocyte ratio; PLR: Platelet to lymphocyte ratio; IG: Immature granulocyte; DNI: Delta neutrophil index; MPV: Mean platelet volume.

IG count and DNI, neutrophil precursor cells can be used as an indicator of bone marrow activation before neutrophil response occurs and can be calculated by automated systems. ${ }^{5}$ In this study, along with the parameters indicative of the inflammatory response, DNI and IG count also increased in axillary metastases before the clinical appearance of axillary metastasis in breast cancer and was found to be a predictive factor.

Neutrophils and macrophages play a role as a stimulating factor in tumor development (for cellular proliferation of the tumor, angiogenesis, invasion, and metastasis). ${ }^{16}$ Proctor et al. found that NLR and PLR were predictive factor of mortality in different cancer types (bladder, breast, colorectal, gastroesophageal, gynecological, prostate, lung and liver cancers) in their studies. ${ }^{17}$ Peng et al. found higher NLR values in breast cancer patients when compared with normal individuals in their studies on breast cancer. ${ }^{3}$ In another study conducted by Atak et al. in breast cancer patients, NLR was found to be statistically significantly higher in patients with nonsentinal lymph node metastasis and found as a predictive factor. ${ }^{8}$ Similarly, in this study, NLR and PLR values were found to be preoperative predictive factors for the detection of axillary metastasis of breast cancer.

Cancer-induced inflammation causes platelet activation, revealing the procarcinogenic and prometastatic activity of platelets. ${ }^{9}$ MPV is used as an early marker of activated platelets and causes an increase in the survival and spread of cancer cells during spread. ${ }^{9}$ In esophageal, breast, hepato- cellular and colorectal cancers, increased MPV values were observed to correlate with poor prognosis. ${ }^{18}$ In their study conducted with invasive breast cancer patients and patients operated on for benign breast diseases, Gu et al. found increased MPV values as a predictive factor before treatment in invasive breast cancer patients. ${ }^{19}$ In accordance with the literature, preoperative MPV value was found to be a predictive factor for axillary metastasis of invasive breast cancer patients in our study.

The determination of the number of IG can be made with manual techniques and automated systems. DNI is demonstrating bone marrow activation earlier than neutrophil response and has been found to be a helpful indicator in the diagnosis and prognosis of different diseases. ${ }^{5,12,20}$ In a study by Unal, the percentage of IG was found to be an important marker in the differentiation of complicated acute appendicitis and uncomplicated acute appendicitis. ${ }^{20}$ Similarly, in another study conducted by Barut et al., it was found to be a predictive factor for early detection of acute pyelonephritis in patients with ureteral stones. ${ }^{12}$ As a result of tumor-related non-infection bone marrow activation, neutrophil precursor cells get involved in the peripheral circulation. In an other study conducted by Bozan et al., DNI was found to be a significant factor in the differentiation of benign nodular goiter and thyroid malignancies. ${ }^{5}$ Additionally another study conducted by Barut et al., DNI was foun to be a predictive factor for renal cell carcinoma. ${ }^{6}$ Similarly, in the preoperative detection of axillary metastasis of breast cancer, inflammation markers obtained from other blood count sections, along with DNI and IG numbers, were found to be predictive factors. 
The most important limiting factor of this study was its retrospective character and being single center experience. Another important limitation was that patients were not evaluated separately in terms of their menopausal periods. In addition, due to the low number of remaining patients, differences in the number of metastatic lymphnodes were not examined in the study. However, the strongest aspect of the study was being the first study that investigating the relationship between the presence of pathological axillary metastases and DNI and IG counts in breast cancer patients without clinical metastasis.

\section{CONCLUSION}

DNI and IG count, may be valuable, are predictive factors with high sensitivity and specificity in detecting axillary metastasis of breast cancers. The inflammatory parameters DNI and IG count, can be studied directly in new automated blood count systems without additional cost and are easily accessible as MPV.

\section{ETHICAL APPROVAL:}

Ethical approval was obtained from Kahramanmaras Sutcu Imam University, Clinical Research Ethics Committee (Date: 13.05.2020; Session No. 2020/09; Decision No. 19).

\section{PATIENTS' CONTENT:}

Informed consents were taken from all patients before the surgery.

\section{CONFLICT OF INTEREST:}

The authors declared no conflict of interest.

\section{AUTHORS' CONTRIBUTION:}

MBB, FMY: Conception, design, acquisition, analysis, interpretation, drafting the work or revising it critically for important intellectual content, final approval of the version to be published.

ITK: Conception, design, drafting or revising it critically for important intellectual content, final approval of the version to be published.

ST, OFB: Acquisition, analysis, interpretation, final approval of the version to be published.

$A A B$ : Analysis, interpretation, drafting the work or revising it critically for important intellectual content, final approval of the version to be published.

\section{REFERENCES}

1. Koc Z, Saglam Z. Determination of the knowledge and the practice of female patients about breast cancer, preventive measures and breast self examination and effectiveness of education. EurJ Breast Heal 2009; 5(1):25-33.

2. Marín Hernández C, Piñero Madrona A, Gil Vázquez PJ, Galindo Fernández PJ, Ruiz Merino G, Alonso Romero JL, et al. Usefulness of lymphocyte-to-monocyte, neutrophil-to-monocyte and neutrophil-to-lymphocyte ratios as prognostic markers in breast cancer patients treated with neoadjuvant chemotherapy. Clin Transl Oncol 2018; 20(4):476-83. doi: 10.1007/s12094-017-1732-0.

3. Peng Y, Chen R, Qu F, Ye Y, Fu Y, Tang Z, et al. Low pretreatment lymphocyte/monocyte ratio is associated with the better efficacy of neoadjuvant chemotherapy in breast cancer patients. Cancer Biol Ther 2020; 21(2):189-96. doi: 10.1080/15384047.2019.1680057.

4. Miklikova S, Minarik G, Sedlackova T, Plava J, Cihova M, Jurisova $\mathrm{S}$, et al. Inflammation-Based scores increase the prognostic value of circulating tumor cells in primary breast cancer. Cancers (Basel) 2020; 12(5):1134. doi: 10.3390/ cancers12051134.

5. Bozan MB, Yazar FM, Kale IT, Yüzbaşıoğlu MF, Boran ÖF, Azak Bozan A. Delta neutrophil ındex and neutrophil-to-lymphocyte ratio in the differentiation of thyroid malignancy and nodular goiter. World J Surg 2021; 45(2):507-14. doi: 10.1007/s00268-020-05822-6.

6. Barut O, Kutlu Demirkol M, Küçükdurmaz F, Şahinkanat T, Resim S. Pre-treatment delta neutrophil ındex as a predictive factor in renal cell carcinoma. J Coll Physicians Surg Pak 2021; 31(2):156-61. doi: 10.29271/jcpsp. 2021.02.156.

7. Singh R, Mishra MK, Aggarwal H. Inflammation, Immunity, and cancer. Mediators Inflamm 2017; 2017: 6027305. doi: 10.1155/2017/6027305.

8. Ibrahim A, Serkan YF, Tuba A, Erol B, Lütfi P. Can neutrophil to lymphocyte ratio be a predictor tool for the non-sentinel lymph node metastasis in breast cancer? Chirurgia (Bucur) 2019; 114(1):83. doi: 10.21614/chirurgia.114.1.83.

9. Li M, Yue C, Fu S, Zhang X, Zhao CJ, Wang R. Platelet volume is reduced in metastasing breast cancer: Blood profiles reveal significant shifts. Cancer Manag Res 2019; 11:9067-72. doi: 10.2147/CMAR.S221976.

10. Song $Y$, Yang $Y$, Gao $P$, Chen $X, Y u D, X u Y$, et al. The preoperative neutrophil to lymphocyte ratio is a superior indicator of prognosis compared with other inflammatory biomarkers in resectable colorectal cancer. BMC Cancer 2017; 17(1): 744. doi: 10.1186/s12885-017-3752-0.

11. Bozan MB, Yazar FM, Güler Ö, Azak Bozan A, Boran ÖF. Preoperative immature granulocyte count and percentage for complicated acute appendicitis and uncomplicated acute appendicitis (A retrospective cohort study). Med Sci 2021; 25(110):760-6.

12. Barut O, Kutlu Demirkol M, Bilecan EB, Sahinkanat T, Resim $\mathrm{S}$. The delta neutrophil Index is an early predictive marker of acute pyelonephritis in patients with ureteral stone. J Coll Physicians Surg Pak 2020; 30(11):1149-54. doi: 10.29271/ jcpsp.2020.11.1149.

13. De Giorgi U, Mego M, Scarpi E, Giordano A, Giuliano M, Valero $\mathrm{V}$, et al. Association between circulating tumor cells and peripheral blood monocytes in metastatic breast cancer. Ther Adv Med Oncol 2019; 11:175883591986606. doi: 10.1177/1758835919866065.

14. Azab B, Shah N, Radbel J, Tan P, Bhatt V, Vonfrolio S, et al. Pretreatment neutrophil/lymphocyte ratio is superior to platelet/lymphocyte ratio as a predictor of long-term mortality in breast cancer patients. Med Oncol 2013; 30(1):432. doi: 10.1007/s12032-012-0432-4.

15. Väyrynen JP, Tuomisto A, Klintrup K, Mäkelä J, Karttunen TJ, Mäkinen MJ. Detailed analysis of inflammatory cell infiltration in colorectal cancer. Br J Cancer 2013; 109(7): 
1839-47. doi: 10.1038/bjc.2013.508.

16. Bozkurt O, Karaca H, Berk V, Inanc M, Ocak Duran A, Ozaslan $\mathrm{E}$, et al. Predicting the role of the pretreatment neutrophil to lymphocyte ratio in the survival of early triplenegative breast cancer patients. JBUON 2015; 20(6): 1432-9.

17. Proctor MJ, Morrison DS, Talwar D, Balmer SM, Fletcher CD, O'Reilly DSJ, et al. A comparison of inflammation-based prognostic scores in patients with cancer. A glasgow inflammation outcome study. Eur J Cancer 2011; 47(17): 2633-41. doi: 10.1016/j.ejca.2011.03.028.

18. Tuncel T, Ozgun A, Emirzeoglu L, Celik S, Bilgi O, Karagoz B.
Mean platelet volume as a prognostic marker in metastatic colorectal cancer patients treated with bevacizumab-combined chemotherapy. Asian Pacific J Cancer Prev 2014; 15(15):6421-3. doi: 10.7314/apjcp. 2014.15.15.6421.

19. Gu M, Zhai Z, Huang L, Zheng W, Zhou Y, Zhu R, et al. Pretreatment mean platelet volume associates with worse clinicopathologic features and prognosis of patients with invasive breast cancer. Breast Cancer2016; 23(5):752-60. doi: 10.1007/s12282-015-0635-6.

20. Ünal Y. A new and early marker In diagnosis of acute complicated appendicitis: Immature granulocyte. Turkish J Trauma Emerg Surg 2018; 24(5):434-9. doi: 10.5505/tjtes. 2018.91661. 